\title{
Análisis de Esquemas de Metadatos para la Marcación de Contenidos Multimedia en Televisión Digital
}

\author{
Angela M. Vargas-Arcila ${ }^{(1)}$, Sandra Baldassarri ${ }^{(2)}$, José L. Arciniegas ${ }^{(1)}$ \\ (1) Universidad del Cauca, CII 5 \# 4-70, Popayán-Colombia. (e-mail: amvargas@unicauca.edu.co, \\ jlarci@unicauca.edu.co) \\ (2) Universidad de Zaragoza, María de Luna 1, 50018 Zaragoza-España. (e-mail: sandra@unizar.es)
}

Recibido May. 7, 2015; Aceptado Jun. 10, 2015; Versión final Jul. 30, 2015, Publicado Dic. 2015

\begin{abstract}
Resumen
Este trabajo presenta un análisis de los principales esquemas de metadatos encontrados en la literatura para la marcación de contenido multimedia de televisión: MPEG-7, TV-Anytime, P-META, EBUCore, PBCore y SMPTE. Además, aclara las características de cada esquema, las relaciones entre ellos, e identifica sus extensiones y perfiles de aplicación para describir contenido de audio, video o audiovisual. Finalmente, se hace una comparación entre los mismos, y una definición de ventajas y desventajas, permitiendo señalar a TV-Anytime y MPEG-7 como los esquemas más aptos para la descripción de recursos multimedia en el contexto de la televisión.
\end{abstract}

Palabras clave: esquema de metadatos; contenidos multimedia; televisión digital; MPEG-7; TV-Anytime; P-META; EBUCore; PBCore; SMPTE

\section{Analysis of Metadata Schemas for Marking Up Multimedia Content in Digital Television}

\begin{abstract}
This paper presents an analysis of the most relevant metadata schemas for marking up multimedia content of television found in the literature: MPEG-7, TV-Anytime, P-META, EBUCore, PBCore and SMPTE. Furthermore, the characteristics and relationships between schemas are clarified, and their extensions and application profiles for audio, video or audiovisual content are identified. Finally, the paper presents a comparison between them identifying advantages and disadvantages that allow defining TVAnytime and MPEG-7 as more suitable than the others for describing multimedia content in television context.
\end{abstract}

Keywords: Metadata scheme; multimedia content; digital television; MPEG-7; TV-Anytime; P-META;

EBUCore; PBCore; SMPTE 


\section{INTRODUCCIÓN}

Los diferentes tipos de servicios posibles en la televisión digital interactiva se clasifican de acuerdo a las características del servicio desde la perspectiva del usuario final en: servicios de distribución de contenido, servicios interactivos, servicios de comunicación y otros servicios (ver fig. 1).

En primer lugar, los servicios de distribución de contenido son los orientados a la difusión de contenido y se clasifican en: servicios de difusión (realizan la transmisión de contenido en una vía hacia uno o más usuarios donde los usuarios tienen un control limitado sobre el contenido, algunos servicios de este tipo son la televisión lineal, servicio de pago por ver, televisión lineal con servicio multivisión, etc.), servicios bajo demanda (le permiten al usuario seleccionar un contenido en cualquier momento), servicios de publicidad, servicios con desplazamiento en tiempo y lugar (el usuario puede acceder y controlar (pausar, rebobinar, adelantar, etc.) su contenido, suscrito en cualquier lugar y sin limitaciones de tiempo), y servicios de contenido complementario (video, audio, texto, gráficos $u$ otros tipos de contenido que pueden ser opcionalmente accedidos por los usuarios, por ejemplo: subtítulos y leyendas, descripción de audio para personas con limitaciones visuales, interpretación en lenguaje de señas, etc.) (ITU-T, 2008).

En segundo lugar se encuentran los servicios interactivos que le permiten al usuario enviar diferentes tipos de peticiones al proveedor de servicios y recibir una realimentación, por ejemplo, los servicios de información que soportan varios tipos de contenido, como noticias, estado del tiempo, etc., servicios comerciales, servicios de entretenimiento diseñados para ofrecer diversión al usuario final (juegos, karaoke, blogs, etc.), servicios de aprendizaje para entregar contenido educativo, servicios médicos, servicios de monitoreo y publicidad interactiva (ITU-T, 2008) (Acevedo et al., 2010). En tercer lugar están los servicios de comunicación que le facilitan la comunicación al usuario final con otros usuarios por medio de mensajería, telefonía, video llamadas o videoconferencias. En cuarto y último lugar se encuentran los servicios que no pueden ser clasificados en ninguna de las categorías anteriores, estos son: servicios de interés público (apoyo a usuarios con discapacidad, comunicación de emergencias, etc.), servicios de alojamiento (por ejemplo: alojamiento de contenido creado por el usuario) y servicios de presencia (manejo del estado del usuario: "viendo la televisión", "viendo un partido de fútbol", etc.) (ITU-T, 2008). Aunque el televidente puede utilizar cualquiera de los servicios anteriores, las dos acciones que los televidentes realizan generalmente son, elegir un programa para verlo en tiempo de difusión o grabar un programa para verlo en otro instante. Para facilitarle al usuario la elección de los contenidos, se necesita información relacionada a ellos, por ejemplo, título, actores, género, resumen, entre otros.

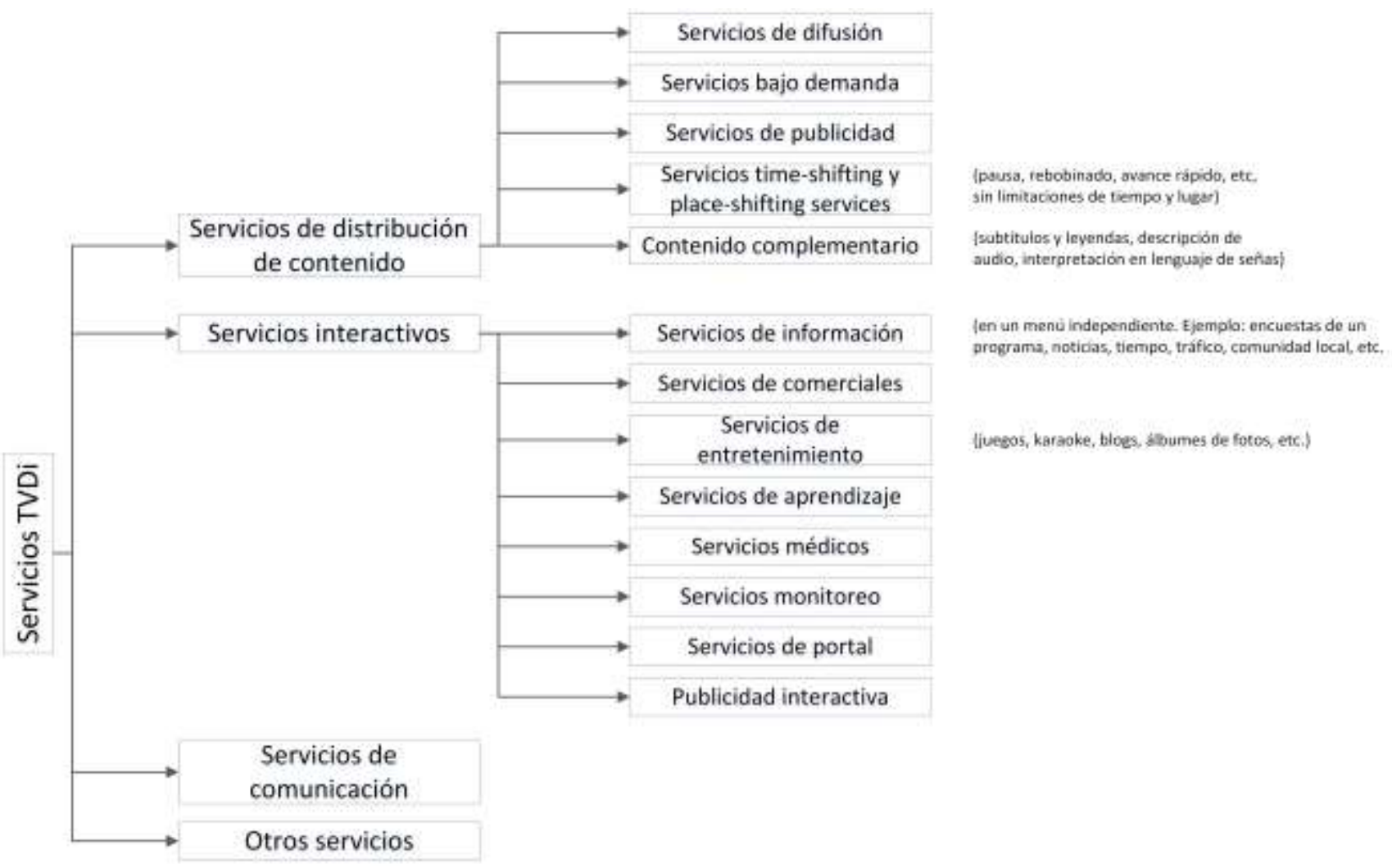

Fig. 1: Clasificación de los servicios de televisión digital interactiva. 
Por su parte, tanto el proveedor de contenidos como el proveedor del servicio de televisión necesitan atraer al usuario hacia sus contenidos proporcionando dicha información; teniendo en cuenta que los datos acerca del contenido que el televidente desea consumir pueden ser almacenados en metadatos, éstos adquieren una gran relevancia (ETSI, 2007). En consecuencia, existen diversos conjuntos de metadatos destinados a la marcación de contenidos de televisión tanto de una manera general como contextualizada, es decir, describen cualquier contenido audiovisual o contenidos particulares como noticias, películas, publicitarios, etc. Dichos conjuntos de metadatos suelen tener una estructura bien definida y un conjunto de reglas para su uso denominada esquema de metadatos.

Existen diversos esquemas de metadatos para la marcación de contenido multimedia de la web y de la televisión, sin embargo, no se ha encontrado en la literatura una recopilación de esquemas que permitan describir este tipo de contenido en el contexto de la televisión, independientemente del ámbito para el que fueron creados, junto con una comparación de sus características e ilustrando las relaciones existentes entre ellos. Por este motivo en este artículo se seleccionan los esquemas de metadatos más relevantes para la marcación de contenido multimedia de televisión con el objetivo de aclarar sus relaciones y características, además de verificar si cumplen con los requerimientos de descripción de contenido de televisión.

\section{ESQUEMAS DE METADATOS EN TELEVISIÓN DIGITAL}

Los metadatos se definen como información que describe, identifica, explica o define un recurso con el objetivo de facilitar su recuperación, uso o gestión. Un metadato es conocido como un dato acerca de otro dato o información sobre información (NISO, 2004). Este trabajo adopta esta definición porque encierra de una manera general el concepto de los metadatos independiente del contexto en donde sean utilizados

Los tipos de metadatos se pueden clasificar de diferentes maneras, por ejemplo, si se tiene en cuenta la función que prestan, éstos se pueden clasificar en metadatos descriptivos (permiten describir un recurso por medio de elementos como título, resumen, autor, palabras clave), metadatos estructurales (indican cómo se componen los recursos que están formados por otros tipos de recursos, por ejemplo: temporada de una serie de televisión, versión de una película, segmento de un programa) y metadatos administrativos (brindan información acerca de las características técnicas del recurso, además de permitir su gestión y administración, por ejemplo: fecha de creación, localización, quién puede acceder a él, formato, tipo de archivo. Existen diversos subtipos de metadatos administrativos de los cuales los metadatos para la gestión de derechos (relacionados con los derechos de propiedad intelectual) y metadatos para la conservación (contienen la información necesaria para almacenar y conservar un recurso) generalmente se presentan como tipos independientes) (NISO, 2004).

Otro tipo de clasificación puede ser la presentada en (Lapuente, 2006) donde los tipos de metadatos se clasifican por su función obteniendo metadatos de acceso (permiten consultar y recuperar el recurso), metadatos semánticos (le dan un significado al recurso), metadatos de calidad (por medio de ellos se puede realizar un análisis cualitativo del recurso), metadatos de transferencia (permiten transferir recursos entre diferentes aplicaciones) y metadatos de almacenamiento (permiten almacenar el recurso).

Por otro lado, existen conjuntos de metadatos que permiten describir un tipo de recurso particular, pues han sido diseñados con una intención concreta, estos conjuntos se conocen como esquemas de metadatos y como parte de su definición también establecen reglas para su uso, por ejemplo, los valores de los metadatos deben estar escritos en mayúscula, o los valores de los metadatos deben ser seleccionados de un vocabulario específico. Un esquema de metadatos puede ser modificado, en primer lugar, por medio de la adición de nuevos metadatos (el resultado se conoce como "extensión"), y en segundo lugar, limitando y refinando el uso de los metadatos existentes, o mezclando metadatos provenientes de diferentes esquemas (el resultado se conoce como "perfil de aplicación") (Vargas-Arcila et al., 2014). En la industria de la televisión digital es común el uso de esquemas de metadatos para describir contenidos de diferentes tipos: películas, noticias, publicidad, etc., y hacer que la gestión e intercambio de estos contenidos, entre diferentes dominios de la cadena de valor de televisión, sea una tarea más sencilla.

Ahora bien, los metadatos que hacen parte de un esquema se conocen como elementos del esquema y usualmente también son denominados como "descriptores", ya que son datos con información descriptiva de un recurso, y como "atrayentes", pues estos datos se utilizan para atraer a los usuarios hacia los recursos de su interés. La cadena de valor general de la televisión digital en cualquiera de sus formas (terrestre, sobre IP, cableada, satelital) está conformada por cuatro dominios: dominio del proveedor de contenidos, dominio del proveedor del servicio de televisión, dominio del proveedor de red y dominio del consumidor (ver fig. 2). En el dominio del proveedor de contenidos se realiza la producción, edición y elaboración de metadatos del contenido (películas, series, eventos, documentales, noticias, etc.). El actor de 
este dominio es el proveedor de contenidos y su rol es desempeñado por una compañía de producción o un distribuidor que tiene licencia para vender contenido y por lo tanto tiene los derechos del recurso audiovisual. En este dominio, los metadatos abarcan toda la información relacionada con las etapas de producción: preproducción, producción y postproducción.

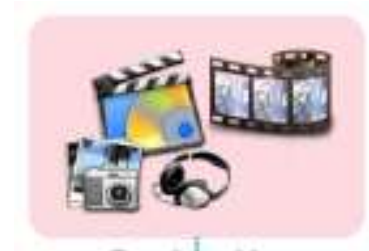

Producción de contenidos

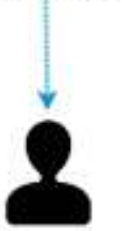

Proveedor de contenidos

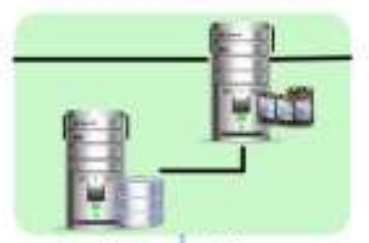

Agregación de contenidos

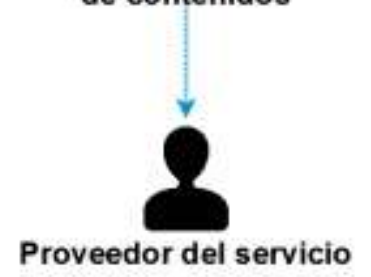

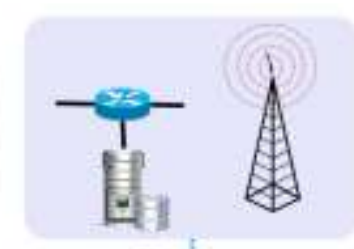

Entrega de contenido

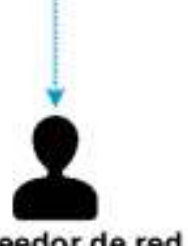

Proveedor de red

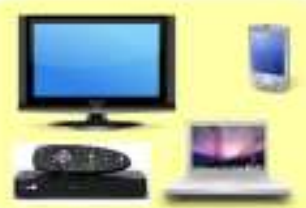

Reconstrucción de contenido

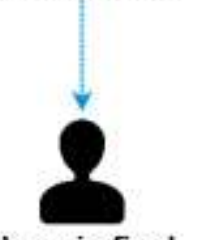

Usuario final

Fig. 2: Cadena de valor de la televisión digital.

En la etapa de preproducción se genera la idea y a partir de ella se elaboran los guiones, el plan de rodaje, la selección de las locaciones, el reparto, el personal artístico y técnico, y el trabajo relacionado con la logística para iniciar la filmación (CINTEL, 2010); algunos ejemplos de los metadatos que reflejan las características de esta etapa son: libretistas, guionistas, creativos. En la etapa de producción se realiza la filmación y por lo tanto se obtiene la mayor parte de las imágenes y sonidos del recurso audiovisual (CINTEL, 2010); algunos de los metadatos que contienen información acerca de esta etapa son: vestuario, maquillaje, escenografía, actores, camarógrafos, técnicos, extras, editores, iluminación, equipos. En la etapa de postproducción se complementa y mejora el contenido por medio de edición, efectos especiales y musicalización (CINTEL, 2010). Algunos de los metadatos correspondientes a esta etapa son: editores, banda sonora, efectos especiales.

De acuerdo a la fig. 2 y siguiendo la cadena de valor, el proveedor de contenidos le proporciona los recursos multimedia al dominio del proveedor del servicio de televisión (CINTEL, 2010) (OIPF, 2013). En el dominio del proveedor del servicio se realiza la agregación de contenido, es decir, se preparan los recursos para ser enviados hacia el usuario final, y se proporcionan los servicios de plataforma (por ejemplo, tarificación, autenticación de usuario, etc.) y los complementos para enriquecer el servicio de televisión (por ejemplo, servicios de comunicación, servicios interactivos, publicidad, etc.). Los contenidos se entregan junto con sus metadatos con el objetivo de facilitar la transferencia y evitar una nueva descripción del recurso por parte del proveedor del servicio de televisión que introduzca errores en la información, sin embargo, el proveedor del servicio de televisión puede crear metadatos principalmente administrativos o, si es necesario, metadatos descriptivos y estructurales relacionados con el contenido. Los metadatos le permiten a este dominio crear adecuadamente una guía de programación y agregarle al usuario servicios que tengan en cuenta la semántica del recurso con el fin de mejorar su experiencia.

Continuando con la cadena de valor, el dominio del proveedor de red se encarga de entregarle el contenido al dominio del consumidor y proporcionar la comunicación entre el dominio del proveedor del servicio de televisión y el dominio del consumidor (OIPF, 2013), quien finalmente está compuesto por el terminal o red de terminales y dispositivos necesarios para el consumo del servicio (televisor, computador, decodificador, Set-Top-Box STB, dispositivo móvil, etc.), dichos dispositivos permiten recibir y reconstruir el contenido a un formato adecuado que pueda ser desplegado por el terminal o dispositivo de usuario final (OIPF, 2013). Es importante resaltar que en la cadena de valor de la televisión digital, no solo se establecen metadatos que describen e identifican los contenidos, sino también el historial o comportamiento del usuario durante su interacción con los servicios de televisión, dichos metadatos se obtienen en este último dominio.

\section{ESQUEMAS DE METADATOS PARA MARCACIÓN DE CONTENIDOS DE TV}

Este trabajo ha considerado diferentes esquemas de metadatos, extensiones y perfiles de aplicación que pueden ser utilizados en la industria de la televisión digital, tales como, SMEF, MXF Metadata Eschema, MPEG-7, EgtaMeta, entre otros, sin embargo, se describen los más destacados y vigentes: MPEG7, TVAnytime, P-META, EBUCore, PBCore y SMPTE Metadata Element Dictionary Structure. 


\section{MPEG-7}

Es un esquema de metadatos para contenido multimedia que permite describir de una manera completa las imágenes digitales, videos digitales o audio digital (Vivancos-Vicente, 2005) (Peig, 2003). Ha sido creado por MPEG (Moving Pictures Experts Group), descrito en el estándar ISO/IEC IS 15938 y nombrado formalmente como Multimedia Content Description Interface. MPEG-7 se enfoca en representar información acerca del contenido y no de la codificación del mismo, como es el caso de los estándares MPEG-1, MPEG2 y MPEG-4 (JISC, s.f a), en otras palabras, MPEG-7 está definido de manera tal que no dependa de cómo el recurso descrito está codificado o almacenado, y por lo tanto, se puede aplicar MPEG-7 para describir recursos que no hacen parte del mundo digital, como por ejemplo una película en soporte analógico (video convencional) o a una imagen impresa sobre un papel (Peig, 2003).

A pesar de que no fue diseñado principalmente para los contenidos de televisión como en el caso de otros estándares, MPEG-7 ofrece un conjunto de metadatos relacionados con la información conceptual de la realidad capturada por el contenido (actores, objetos, eventos) y es capaz de soportar un gran grupo de aplicaciones pues tiene un enfoque genérico y en su creación se consideraron diferentes estándares desarrollados por otros grupos que están orientados hacia aplicaciones concretas, por ejemplo, SMPTE, Dublin Core y EBUCore (Delgado et al, 2006). Además de los metadatos generalmente utilizados para describir contenido multimedia directamente relacionados con el proceso de producción (título, ubicación, actores, etc.), los formatos de almacenamiento y derechos de autor, MPEG-7 permite añadir información semántica a la descripción del contenido (quién, qué, cuándo, dónde, eventos, objetos, etc.), información estructural de bajo nivel (formas, colores, texturas, movimientos, sonidos, etc.), e información relacionada con la estructura espacial, temporal o espacio-temporal (cortes de escena, segmentación en regiones y seguimiento de región en movimiento) (JISC, s.f a).

La información relacionada con la estructura temporal representa una ventaja de MPEG-7, pues significa que tiene la capacidad de segmentar, en el tiempo, el contenido y asignarle diferentes metadatos a cada parte (JISC, s.f a), además de permitir definir diferentes tipos de segmentos y crear jerarquías de segmentos (Rey-López et al., 2010). Por otro lado, sus metadatos no se presentan en forma de lista, sino que hacen parte de una estructura jerárquica diseñada por medio de agrupaciones de metadatos, como se representa en la fig. 3.

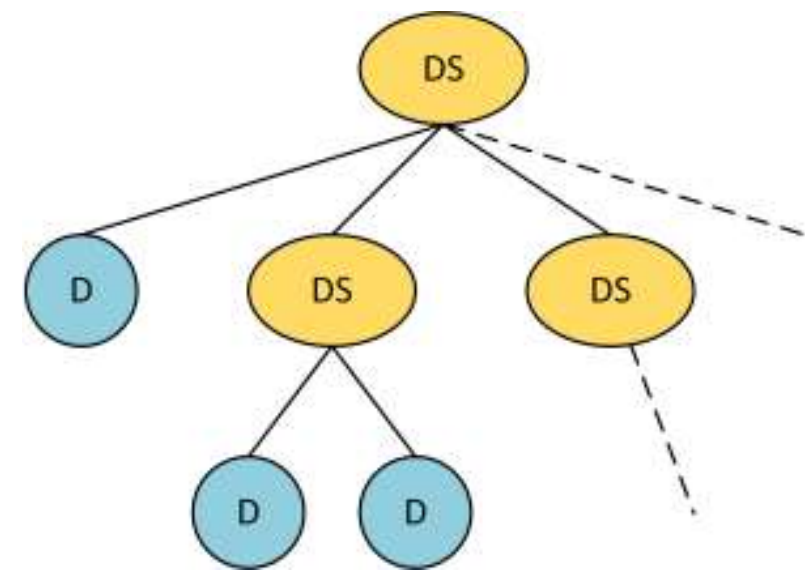

Fig. 3: Representación de la estructura de MPEG-7 (Rey-López, 2009).

En la fig. 3 los metadatos representados por la letra "D", son llamados descriptores, pues como se dijo anteriormente, se utilizan para incluir la información relacionada con los procesos de creación y producción del contenido, su uso, su estructura (espacio-temporal) e información descriptiva de lo que aparece en el contenido, es decir, son la representación de una característica del contenido (Peig, 2003). Los descriptores se agrupan de acuerdo a su función para hacer parte de un esquema de descripción o DS (Descripction Scheme) que a su vez especifica tanto la estructura como la semántica de las relaciones entre sus componentes, dichos componentes pueden ser descriptores (D) o esquemas de descripción (DS).

Con el objetivo de implementar los esquemas de descripción, sus descriptores, los atributos de cada uno de los elementos (DS O D), las relaciones existentes entre ellos, los tipos de datos utilizados (primitivos y compuestos) y los valores posibles de un descriptor, MPEG-7 utiliza el Lenguaje de Definición de Descriptores o DDL (Description Definition Language), el cual está basado en XML y que permite la extensión y modificación de los elementos existentes (Peig, 2003). La fig. 4 muestra la relación entre los diferentes elementos MPEG-7. 


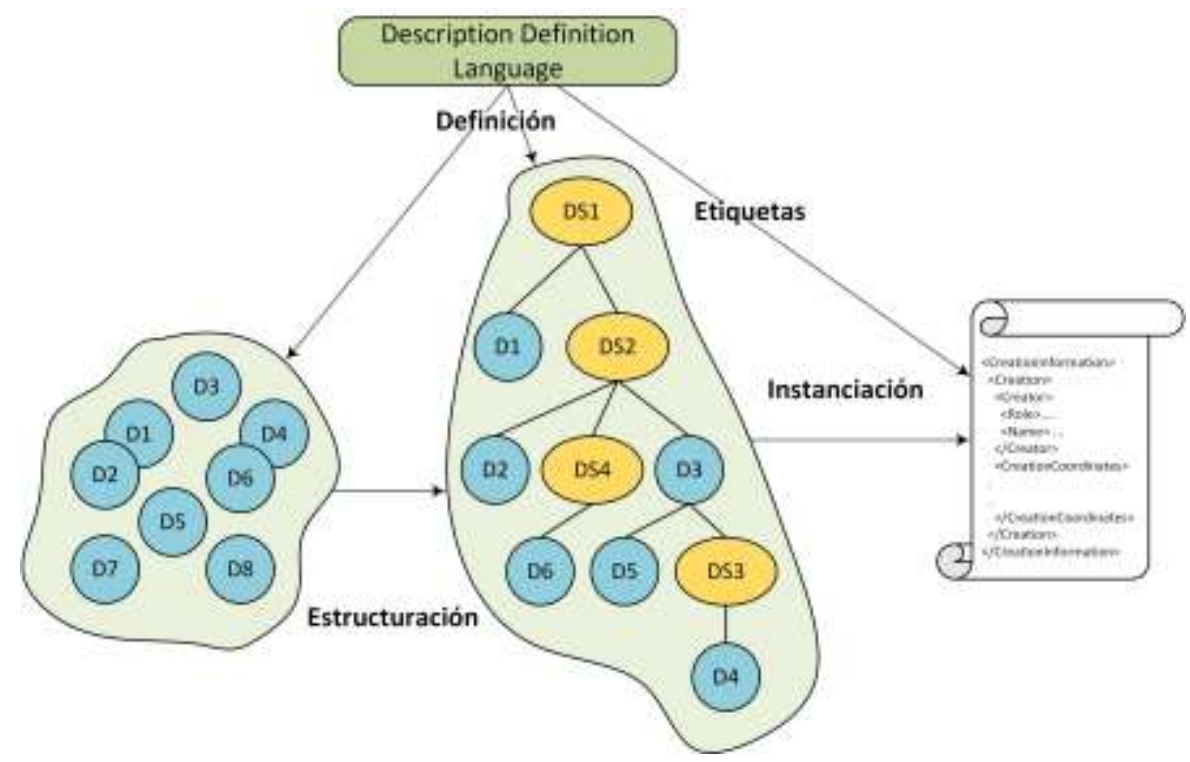

Fig. 4: Relación de los elementos principales de un sistema mpeg-7 (Peig, 2003).

MPEG-7 también utiliza el concepto de esquemas de clasificación (Classification Scheme CS). Un esquema de clasificación es una lista de términos definidos y con su significado, de esta manera, las herramientas de descripción que implementan MPEG-7 pueden utilizar los términos que ya han sido organizados por los CS para asignarle un valor a metadatos particulares (Feng et al., 2003).

\section{TV-Anytime}

TV-Anytime (TVA) es un estándar publicado por medio de reportes y especificaciones técnicas de la ETSI (ETSI TS 102323 y las series de ETSI TS 102 822) que son referenciados dentro de las recomendaciones ITU. Su creador es TV-Anytime Forum, un consorcio mundial dedicado a producir estándares para los receptores de televisión y liderado por los fabricantes de electrónica de consumo, difusores, operadores de telecomunicaciones, etc. de todo el mundo (Delgado et al, 2006). El conjunto de especificaciones que abarca TVA tiene como objetivo permitir la búsqueda, selección, adquisición y uso correcto de contenido en sistemas de almacenamiento personales o locales desde servicios en línea o de difusión, de esta manera TVA pretende ser una guía para los fabricantes y proveedores del servicio de televisión (ETSI, 2006). El estándar se desarrolló en dos fases y tiene en cuenta el uso de metadatos para cumplir con sus objetivos, por eso, TVA define claramente un esquema de metadatos para contenidos audiovisuales, el cual ha heredado su nombre.

Los metadatos definidos en TV-Anytime para describir el contenido se basan en la hipótesis: "un metadato es un dato descriptivo acerca del contenido que el usuario quiere consumir", por lo tanto gran parte de sus metadatos son atrayentes (ETSI, 2007). En la primera fase de TVA, se definen los metadatos que permiten describir el contenido audiovisual, proporcionar información de segmentación del contenido y grupos de segmentos, describir las preferencias del usuario o historial de consumo (metadatos obtenidos a la hora de consumir el material multimedia), agregar información acerca del proveedor de metadatos junto con sus derechos, establecer políticas relacionadas con los derechos y privacidad del recurso (ETSI, 2007) (ReyLópez, 2009). La fig. 5 muestra los elementos principales de TVA fase 1.

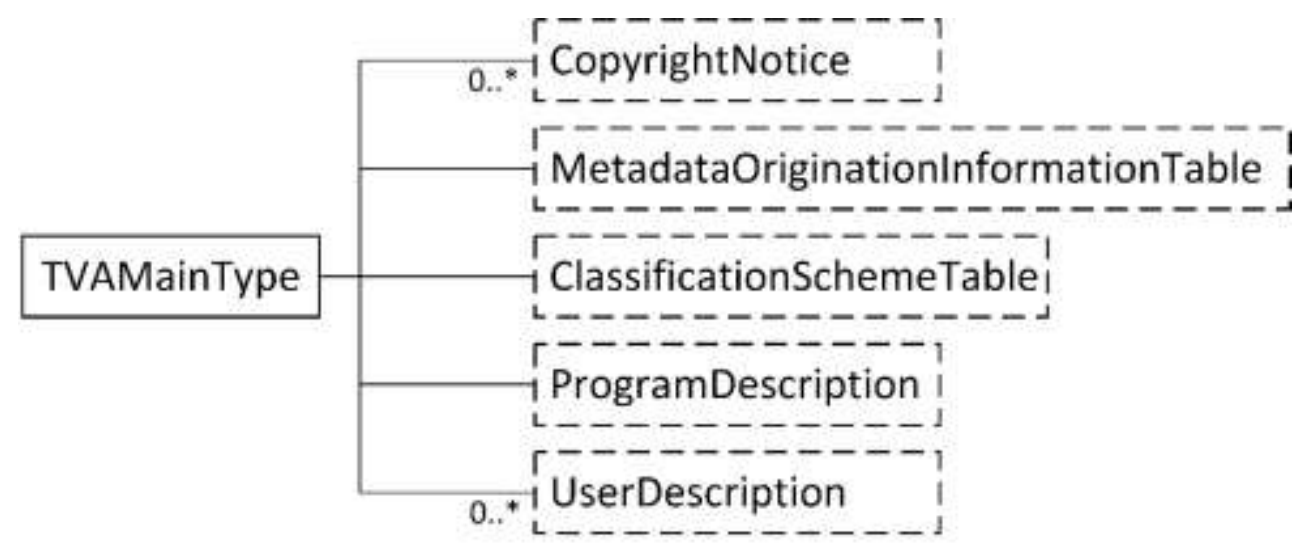

Fig. 5: Elementos principales de TVA fase 1 (ETSI, 2012a). 
La segunda fase de TVA amplía la fase 1 para cubrir todos los datos descriptivos acerca del contenido y también datos acerca de cómo se debe consumir el contenido. Entre los nuevos elementos que incluye se encuentran: empaquetamiento de contenido, metadatos de focalización, capacidades del dispositivo y nuevos tipos de contenido (ETSI, 2012b).

En la televisión digital, un paquete es una colección de elementos que están destinados a ser consumidos de manera conjunta para brindar diversas experiencias al usuario, por ejemplo, es posible tener un curso audiovisual de francés acompañado de un juego de palabras que ayudará a aprender mejor este idioma. Lo anterior es posible gracias a la definición del empaquetado en la especificación TS 102 822-3-3 de TVA que permite la combinación de diferentes tipos de elementos de contenido, tales como juegos, aplicaciones, imágenes y texto. Dentro de esta definición, es importante resaltar que los nuevos metadatos introducidos en TVA fase 2 permiten describir los nuevos tipos de contenido además de la información de sincronización temporal y espacial entre los elementos del paquete para permitir que el recurso sea consumido como lo previó el creador del contenido. Gracias a la información de sincronización, se puede proporcionar experiencias multiflujo con los paquetes de contenido (por ejemplo: un partido desde diferentes ángulos (multicámara)) (ETSI, 2007). Por otro lado, una de las características relevantes que introduce TVA fase 2 es la posibilidad de utilizar metadatos que reflejen las características del enfoque del contenido, es decir, el tipo de usuario a quien va dirigido, el dispositivo que puede soportarlo, etc., y de esta manera le garantiza a agentes software (una aplicación en el dispositivo final o un servicio del proveedor), entregar el contenido relevante de acuerdo a un determinado perfil de usuario o historial de uso que existe en el dispositivo final de usuario o en un servidor (ETSI, 2007).

Otro aspecto importante en el contexto de la televisión es la gestión de los derechos y la protección de información RMPI (Rights Management \& Protection Information), elementos que incluye TVA fase 2 para permitirle al usuario conocer los derechos asociados al contenido antes de que lo adquiera. Los nuevos elementos principales de TVA incluidos en la fase 2 se muestran en la fig. 6.

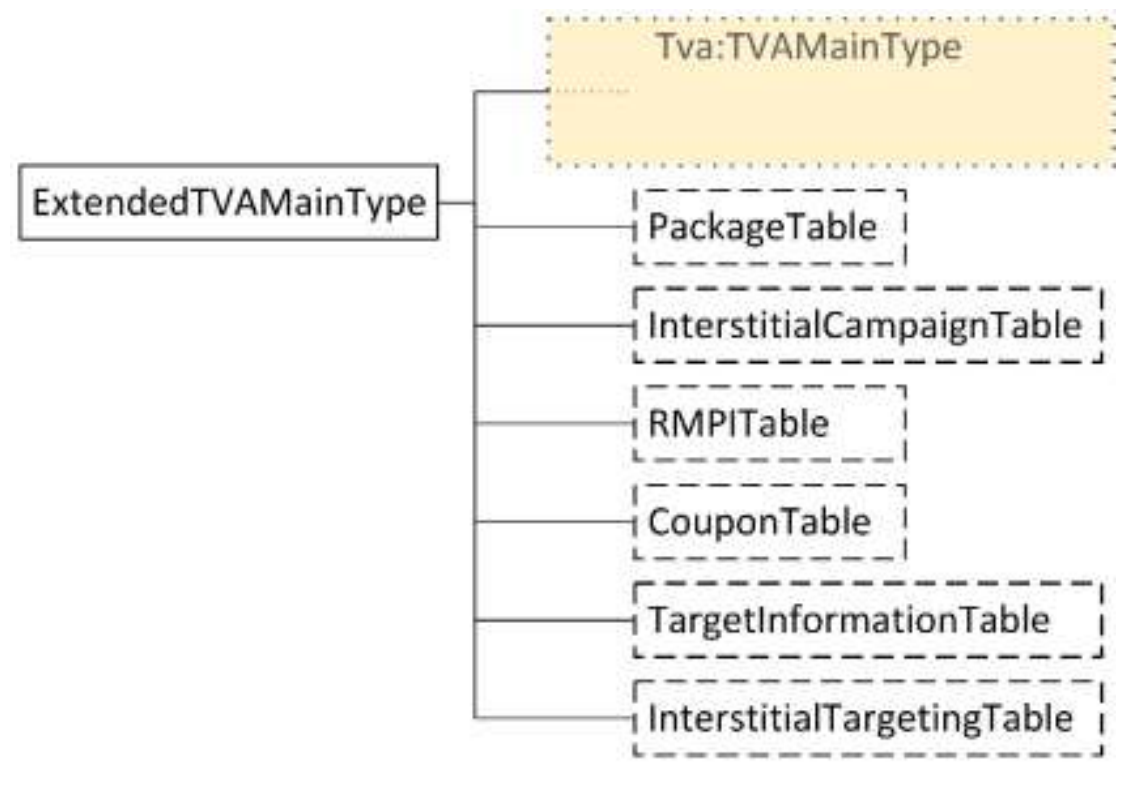

Fig. 6: Elementos principales incluidos en TVA fase 2 (ETSI, 2012b).

La descripción del contenido por medio de TVA es totalmente independiente del canal, horario y protocolo de difusión utilizado, para garantizar esta independencia, el estándar propone asociar a cada contenido un identificador único denominado CRID (Content Referente IDentifier) para que pueda ser referenciado (ReyLópez, 2009). Al igual que MPEG-7 ha escogido a DDL como su formato de representación de metadatos (Delgado et al, 2006) y utiliza la misma filosofía de esquemas de clasificación para escoger un valor para un metadato dentro de un conjunto de posibles valores ya definidos.

Este estándar permite describir contenidos audiovisuales y segmentos de contenido, permitiendo la navegación dentro de una pieza de contenido segmentado, permite describir paquetes de contenidos que de alguna manera se combinarán para ser presentados en conjunto, además tiene un conjunto de metadatos destinado a describir las preferencias del usuario y hábitos de consumo que pueden ser utilizados por aplicaciones o agentes software que buscan y seleccionan contenido apropiados para el usuario (Delgado et al, 2006) (TV-Anytime Forum, 2003) (JISC, s.f b). 


\section{P-META}

Desde 1999 el grupo ECM MAG (Expert Community on Metadata - Multistakeholder Advisory Group) del proyecto EBU (European Broadcasting Union) trabaja en la creación de un vocabulario estándar para la información relacionada con los productos audiovisuales en la industria de difusión audiovisual y ha diseñado el esquema de metadatos semántico EBU P-META (EBU P-Meta Semantic Metadata Schema), conocido como P-META definido en la especificación EBU TECH 3295 (EBU Technical Specification Tech 3295). Este esquema se creó originalmente para soportar el intercambio de contenidos entre organizaciones o sistemas de producción, pero también se ha utilizado como un conjunto de metadatos semánticos descriptivos (EBU, 2011).

El objetivo inicial consistió en un esquema de metadatos común con diferentes proyectos que se desarrollaron con el mismo fin como SMPTE, MPEG-7, Dublin Core y TV-Anytime, pero esta armonización fue imposible debido a los diferentes intereses detrás de cada uno de ellos (Delgado et al, 2006). Por esta razón, P-META se traslapa en diferentes aspectos con otros conjuntos de metadatos desarrollados por diferentes organizaciones de la industria (EBU, s.f). Actualmente se encuentra en su versión 2.2 y publicado para su uso libre (EBU, s.f).

P-META es una lista plana de metadatos centrada en el intercambio de productos audiovisuales comerciales entre difusores (Marcos, 2011). También se compone de un conjunto de tipos de datos, reglas sintácticas y una librería de términos controlados (definidos en listas de clasificación para favorecer la interoperabilidad y permitir su traducción a diferentes idiomas (EBU, s.f) que se deben tener en cuenta en el momento de generar los metadatos que identificarán editorialmente o describirán técnicamente un recurso específico y sus derechos asociados (EBU, 2011) (Marcos, 2011).

Desde el punto de vista tecnológico, P-META ha sido diseñado para ser lo más flexible posible en la implementación, pues no va más allá de la definición de los términos y por lo tanto puede ser materializado de por medio de diferentes maneras: como documentos XML, plantillas de Word e insertando metadatos en formatos de archivos como MXF (SMPTE 377-1-2009 Material Exchange Format) o BWF, de esta manera, P-META es independiente de la tecnología (EBU, 2011) (Marcos, 2011). P-META puede ser implementado tal y como está definido en su especificación o puede ser extendido para cubrir necesidades específicas (EBU, 2011) (EBU, s.f). Sin embargo, P-META no está destinado para ser usado en bases de datos (aunque podría ser utilizado como un punto de partida) (EBU, s.f).

\section{EBUCore}

El grupo ECM MAG del proyecto EBU definió a EBUCore en la especificación EBU TECH 3293 (EBU Technical Specification Tech 3293) como un esquema de metadatos basado en Dublin Core con el objetivo de maximizar su interoperabilidad con la comunidad de usuarios de este último y por esta razón se conoce como el Dublin Core para multimedia (EBU, 2014). Es un conjunto de metadatos descriptivos, administrativos técnicos y estructurales que permiten describir un contenido audiovisual con la mínima información necesaria y que más allá de permitir la descripción del contenido en entornos de producción, también puede ser usado para describir características de distribución de ese contenido (EBU, 2014).

EBUCore ha sido diseñado con una mínima y flexible lista de metadatos para describir recursos de audio y video para una amplia gama de aplicaciones de difusión incluyendo intercambio de recursos y sistemas distribuidos, además cuenta con una sintaxis y semántica bien definida que facilita su implementación (EBU, 2014). Actualmente se encuentra en su versión 1.5 la cual tiene en cuenta los últimos desarrollos de la Web Semántica y las comunidades Linked Open Data, razón por la cual EBUCore 1.5 está disponible en RDF.

La caracterización y semántica de cada metadato de EBUCore se define a por medio de la siguiente estructura (EBU, 2014):

a) Nombre (Name): Es el nombre del metadato. Por ejemplo: fileSize es el nombre del metadato que permite ingresar el tamaño del archivo correspondiente al recurso que se está describiendo.

b) Cardinalidad (Cardinality): Es el número de veces que un metadato puede ser utilizado cuando describe una característica del contenido. Por ejemplo: siguiendo con el ejemplo anterior, la cardinalidad de fileSize es de 0 a 1, es decir puede aparecer máximo una vez en la descripción del recurso o no utilizarse.

c) Requerimiento (Requirement): Indica si se requiere el metadato o es opcional.

d) Definición (Definition): Proporciona una corta descripción del metadato y su uso. 
e) Formato (Format): Define el tipo de formato del metadato. Por ejemplo: un tipo complejo, texto o URI.

f) Esquema (Schema): Muestra una vista sintáctica de la representación del metadato en el esquema EBUCore.

g) Representación UML

h) Semántica para cada metadato y sus atributos con referencia a datos y ejemplos.

EBUCore se rige por la licencia Attribution-NonCommercial-ShareAlike3.0 Unported (CC BY-NC-SA 3.0) de Creative Commons, y por lo tanto le permite al usuario adaptar el esquema a sus necesidades particulares por medio de extensiones que utilicen definiciones propietarias que no entren en conflicto con EBUCore y que pueden ser utilizadas dentro de sus dominios de aplicación. Sin embargo, el usuario debe responsabilizarse por compartir las definiciones de sus extensiones con terceros para permitir su interoperabilidad (EBU, 2014).

Algunos de los perfiles de aplicación basados en EBUCore son EBU ADM (EBU Audio Definition Model) y EgtaMETA. EBU ADM está descrito en la especificación EBU TECH 3364 (EBU Technical Specification Tech 3364) y proporciona un completo conjunto de metadatos técnicos e informativos para describir archivos con contenido de audio. Actualmente ya está incorporado en la especificación 3293 y por lo tanto es parte de su esquema base EBUCore. EgtaMETA, por su parte, está descrito en la especificación EBU TECH 3340 (EBU Technical Specification Tech 3340), define la sintaxis y semántica de descripciones estructuradas interpretables por máquina para la descripción e intercambio de material publicitario (EBUEGTA, 2010). Por último, es importante resaltar que actualmente EBUCore ha reemplazado a la especificación P-META, descrita anteriormente.

\section{PBCore}

PBCore (Public Broadcasting Metadata Dictionary) es un esquema de metadatos de uso libre, financiado por la Corporación para la Difusión Pública de Estados Unidos (Corporation for Public Broadcasting) (PBCore Project, 2011a). Está diseñado para ser utilizado por los proveedores de televisión, radio y web, y pretende ser una manera estándar de describir y usar el contenido multimedia con el objetivo de facilitar la recuperación y compartición de recursos entre colegas, sistemas software, instituciones, socios de producción, particulares, etc. (JISC, s.f a). Desde su lanzamiento en 2005, ha sido adoptado por muchos usuarios que hacen parte de la industria audiovisual y actualmente se encuentra en su versión 2.0 (PBCore Project, 2011a).

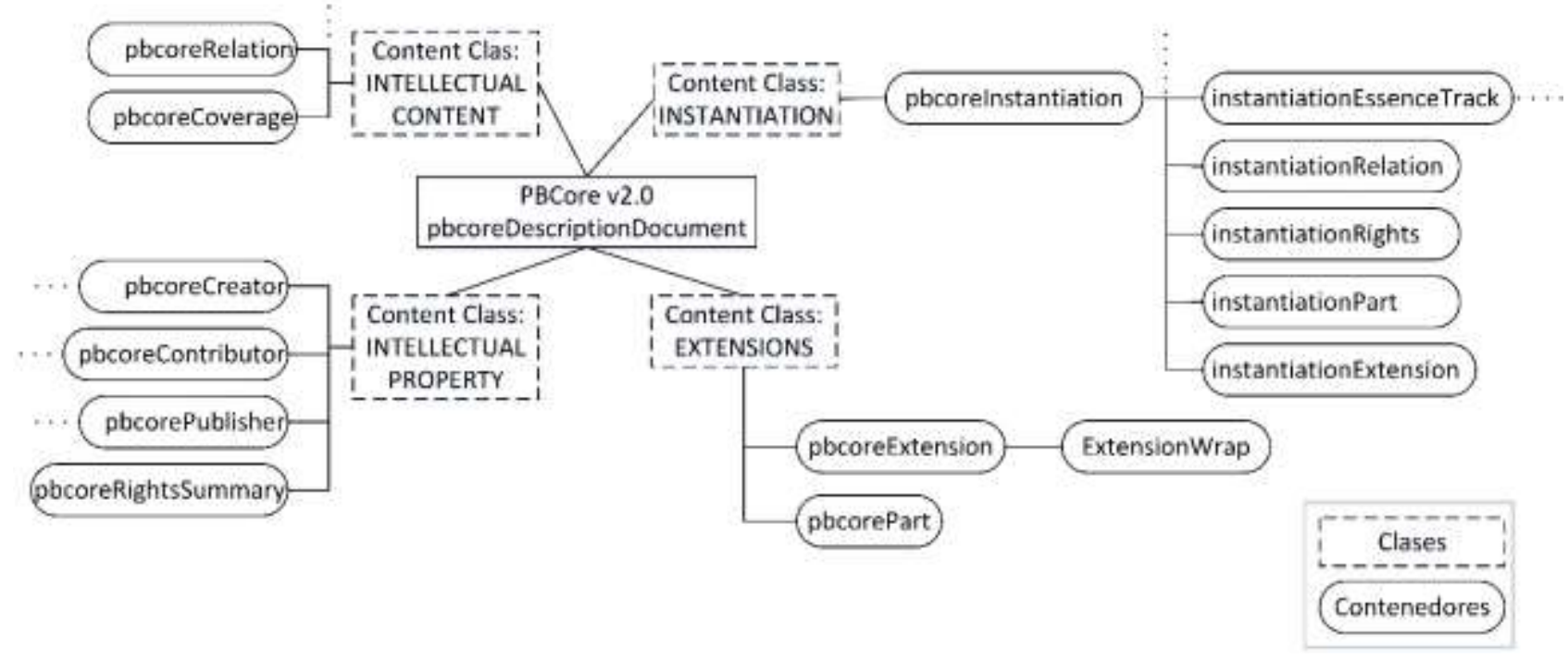

Fig. 7: Estructura de PBCore (PBCore Project, 2011c).

Se basa en Dublin Core y añade un número de metadatos útiles para multimedia obteniendo como resultado una gran cantidad de metadatos organizados en 15 contenedores que a su vez se clasifican en 4 clases (ver fig. 7) (PBCore Project, 2011b):

1.- PBCorelntellectualContent: Metadatos que describen el recurso audiovisual (título, género, descripción, etc.). 
2.- PBCorelntellectualProperty: Metadatos relacionados con la creación del recurso (creador, permisos de uso, restricciones, etc.).

3.- PBCorelnstantiation: Metadatos que identifican la naturaleza del recurso (tamaño del archivo, tipo de archive, etc.)

4.- PBCoreExtensions: Descripciones adicionales que han sido diseñadas por organizaciones ajenas al Proyecto PBCore.

Al igual que Dublin Core tiene la intención de ser simple y ser un punto de partida para que los usuarios lo tomen como base en la creación de sus propias extensiones (JISC, s.f a).

PBCore también puede ser utilizado como modelo de datos para sistemas de catalogación de medios o gestión de archivos pues está organizado como un conjunto de campos que pueden ser utilizados en aplicaciones con bases de datos (PBCore Project, 2011a).

\section{SMPTE Metadata Element Dictionary Structure}

SMPTE (Society of Motion Picture and Television Engineers) es una organización internacionalmente reconocida en el desarrollo de estándares relacionados con imagen, sonido y metadatos; su estándar SMPTE ST 335 (Metadata Element Dictionary Structure) define un extenso y complejo esquema de metadatos para recursos audiovisuales y está diseñado para cubrir toda la cadena de producción (Delgado et al, 2006) (SMPTE, 2012).

Los metadatos definidos por SMPTE se clasifican en varias clases de metadatos estructuradas de forma jerárquica (ver fig. 8). Las clases de metadatos con conjuntos de metadatos con características y atributos comunes. Esta clasificación facilita la gestión de los metadatos, proporciona flexibilidad en su captura e intercambio de metadatos entre aplicaciones (SMPTE, 2012). Las quince clases base de la jerarquía son (SMPTE, 2012):

Clase 1: Identificación y ubicación (identification and location): Los metadatos la información de identificación del contenido.

Clase 2: Administración (administrative): Los metadatos en esta clase están compuestos de datos administrativos, por ejemplo, información acerca de la autorización, restricciones de uso y cifrado. La información de costo e información necesaria para proteger la propiedad intelectual también son elementos que contiene esta clase. Algunos ejemplos de nombres de metadatos en esta clase son: supplier (proveedor), rights (derechos), financial information (información financiera), security (seguridad), participating parties (partes participantes), broadcast and repeat statistics (estadísticas de difusión y repetición).

Clase 3: Interpretativo (interpretive): Los metadatos de esta clase contienen la información descriptiva, por ejemplo, palabras clave, sinopsis, títulos, géneros, etc. Esta información se utiliza principalmente para indexación, catalogación, administración, búsqueda y recuperación del contenido.

Clase 4: Paramétrico (Parametric): En esta clase los metadatos contienen la información que describe las características técnicas de la cámara o sistema que origina el contenido.

Clase 5: Proceso (Process): Los metadatos en esta clase tienen información que describe cómo se procesó el recurso o cómo se cambió o mejoró después de su creación.

Clase 6: Relacional (Relational): Los metadatos de esta clase contienen la información que describe las relaciones entre objetos en el contenido.

Clase 7: Espacio-Temporal (Spatio-temporal): Los metadatos tienen la información acerca de aspectos relacionados con el tiempo, lugar o espacio. La información geoespacial en la clase 7 es cualquier información que defina los lugares o posiciones de objetos, escenas, individuos, o cualquier otro componente del contenido.

Clase 13: Registrados para uso público (Organizationally registered for public use): Esta clase contiene metadatos individuales que han sido registrados por una organización específica y por lo tanto, son reservados y gestionados por separado de las otras clases de metadatos (1 a 7) 
Clase 14: Registrados como privados (Organizationally registered as private): Esta clase contiene metadatos individuales cuyas definiciones están en manos de una organización específica y por lo tanto, están reservados y gestionados por separado de otras clases de metadatos (1 a 7).

Clase 15: Experimental: Esta clase solo debe ser utilizada en un contexto de investigación o en entornos experimentales donde la experimentación con nuevos elementos de metadatos y aplicaciones no dependan estrictamente de su conformidad con los estándares existentes y se mantengan dentro de un entorno de prueba o laboratorio.

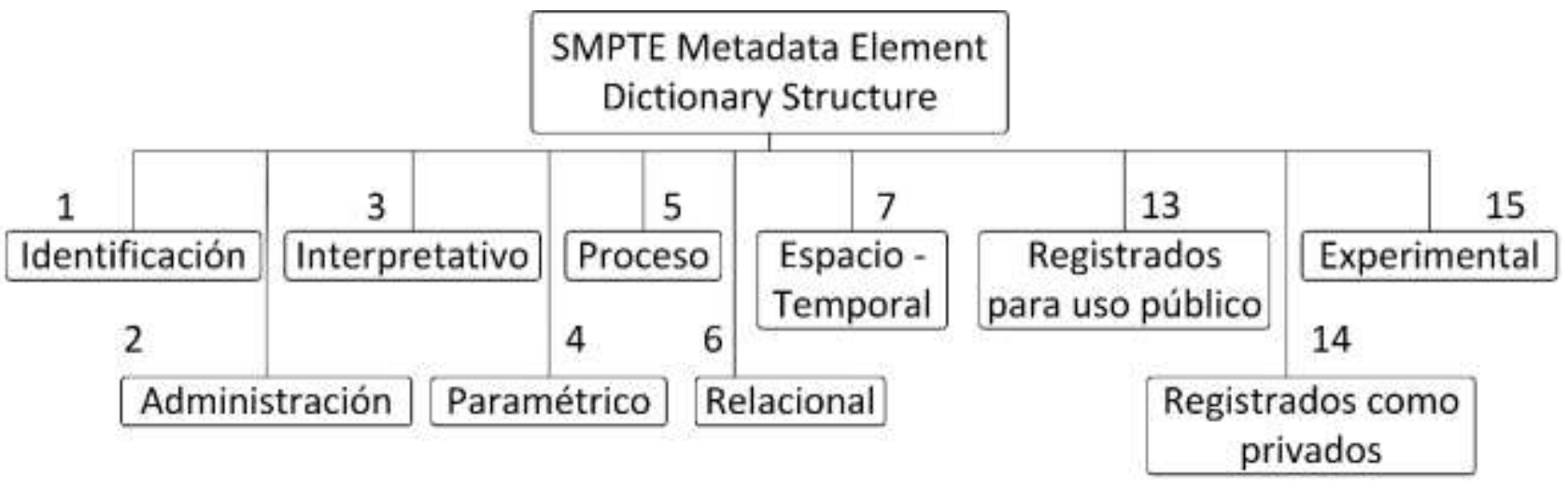

Fig. 8: Estructura de SMPTE (SMPTE, 2012).

\section{RELACIÓN Y COMPARACIÓN ENTRE ESQUEMAS}

En la fig. 9 se muestra gráficamente las relaciones que existen entre los estándares estudiados, resaltando a los que tienen mayor repercusión en el contexto de la televisión

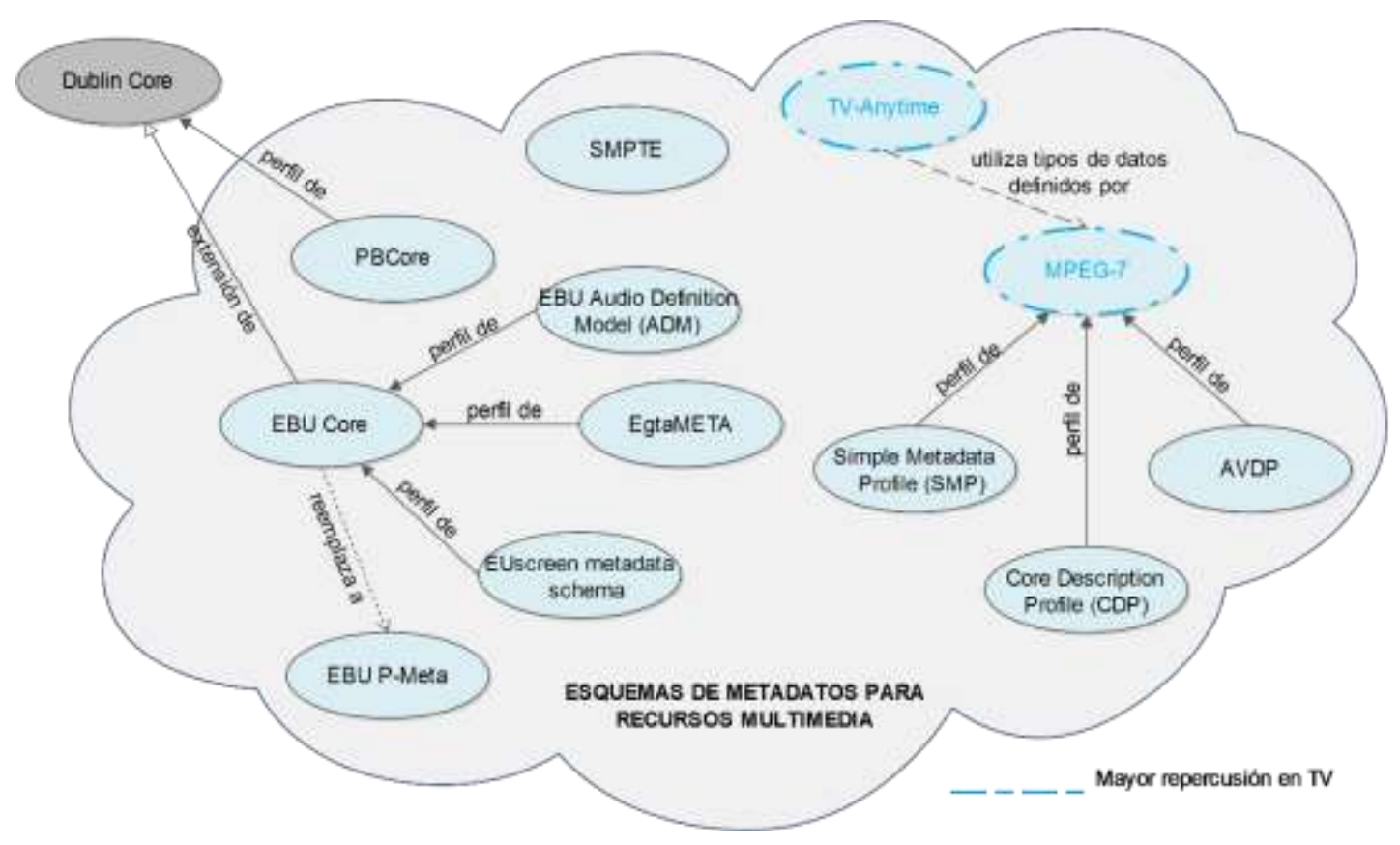

Fig. 9: Relación entre esquemas de metadatos para contenidos multimedia en televisión.

La ¡Error! La autoreferencia al marcador no es válida. muestra una comparación entre los anteriores esquemas de metadatos por medio de cinco elementos. En primer lugar, se presenta su creador junto con el nombre del estándar o especificación y su enlace. En este punto es importante aclarar que una especificación es una propuesta de organismos que no ha sido aprobada por algún organismo oficial de estandarización, mientras que un estándar es una tecnología, formato o método ratificado por algún organismo oficial de estandarización, en algunos casos, una especificación puede considerarse un estándar de facto si su uso es extendido y entretanto se ratifica como estándar. 
Tabla 1: Características de los esquemas de metadatos para contenidos multimedia en televisión

\begin{tabular}{|c|c|c|c|c|c|c|}
\hline \multirow{2}{*}{ Nombre } & \multirow{2}{*}{$\begin{array}{l}\text { Desarrollado por/ } \\
\text { enlace lestándar o } \\
\text { especificación }\end{array}$} & \multirow{2}{*}{$\begin{array}{c}\text { Esquema de } \\
\text { metadatos } \\
\text { base }\end{array}$} & \multirow{2}{*}{$\begin{array}{l}\text { Permite } \\
\text { extensiones }\end{array}$} & \multicolumn{2}{|c|}{$\begin{array}{c}\text { Perfiles/extensiones } \\
\text { Relacionados con multimedia }\end{array}$} & \multirow{2}{*}{$\begin{array}{l}\text { Seg } \\
\text { Mentá } \\
\text { Ción }\end{array}$} \\
\hline & & & & Nombre & $\begin{array}{l}\text { Aplicá } \\
\text { Ción }\end{array}$ & \\
\hline \multirow{4}{*}{ MPEG-7 } & \multirow{4}{*}{$\begin{array}{l}\text { Desarrollado Por: } \\
\text { Moving Picture } \\
\text { Coding Experts } \\
\text { Group (Mpeg) } \\
\text { Enlace: } \\
\text { Http://Www.Iso.Org/ } \\
\text { Estándar: } \\
\text { Iso/lec } 15938\end{array}$} & & \multirow{4}{*}{$\begin{array}{l}\text { Sí } \\
\text { (principalmente } \\
\text { por medio de } \\
\text { esquemas de } \\
\text { clasificación) }\end{array}$} & \begin{tabular}{|l} 
Audio Visual \\
Detailed Profile \\
(AVDP)
\end{tabular} & $\begin{array}{l}\text { audio, video } \\
\text { contenido } \\
\text { audiovisual } \\
\end{array}$ & \multirow{4}{*}{$\begin{array}{c}\text { en tiempo y } \\
\text { espacio }\end{array}$} \\
\hline & & & & $\begin{array}{l}\text { Simple } \\
\text { Metadata } \\
\text { Profile (SMP) }\end{array}$ & multimedia & \\
\hline & & & & \begin{tabular}{|l} 
Core \\
Description \\
Profile (CDP)
\end{tabular} & multimedia & \\
\hline & & & & PrestoSpace & noticias & \\
\hline TVA & $\begin{array}{l}\text { Desarrollado Por: } \\
\text { Tv-Anytime Forum } \\
\text { Enlace: } \\
\text { Http:/Www.Tv- } \\
\text { Anytime.Org/ } \\
\text { Estándar: } \\
\text { Etsi Ts } 102822\end{array}$ & & \begin{tabular}{|c|} 
Sí \\
(principalmente \\
por medio de \\
esquemas de \\
clasificación)
\end{tabular} & & & $\begin{array}{l}\text { en tiempo } \\
\text { (descripción } \\
\text { limitada) }\end{array}$ \\
\hline P-META & $\begin{array}{l}\text { Desarrollado Por: } \\
\text { Ebu Project Group } \\
\\
\text { Enlace: } \\
\text { https://tech.ebu.ch/m } \\
\text { etadata/p meta } \\
\text { Especificación: } \\
\text { Tech } 3295 \\
\text { (Septiembre de 2011) }\end{array}$ & & sí & $\begin{array}{l}\text { EBU Music } \\
\text { Reporting } \\
\text { Metadata } \\
\text { EBU TECH } \\
3332\end{array}$ & música & $\begin{array}{l}\text { en tiempo } \\
\text { (descripción } \\
\text { limitada) }\end{array}$ \\
\hline \multirow{3}{*}{ EBUCore } & $\begin{array}{l}\text { Desarrollado por: } \\
\text { EBU Project Group } \\
\text { Enlace: }\end{array}$ & & & \begin{tabular}{|l} 
Audio Definition \\
Model (ADM) \\
EBU TECH \\
3364 \\
\end{tabular} & audio & \multirow{3}{*}{ en tiempo } \\
\hline & $\begin{array}{l}\text { https://tech.ebu.ch/M } \\
\text { etadataEbuCore }\end{array}$ & $\begin{array}{l}\text { Extensión de: } \\
\text { Dublin Core }\end{array}$ & sí & \begin{tabular}{|l} 
EgtaMETA \\
EBU TECH \\
3340 \\
3340
\end{tabular} & publicidad & \\
\hline & $\begin{array}{l}\text { Especificación: } \\
\text { TECH } 3293 \text { (Abril de } \\
\text { 2014) }\end{array}$ & & & $\begin{array}{l}\text { EUscreen } \\
\text { metadata } \\
\text { schema }\end{array}$ & $\begin{array}{l}\text { contenido } \\
\text { audiovisual } \\
\text { para web tv }\end{array}$ & \\
\hline PBCore & $\begin{array}{l}\text { desarrollado por: } \\
\text { corporation for public } \\
\text { broadcasting } \\
\text { enlace: } \\
\text { http://www.pbcore.org/ } \\
\text { estándar: } \\
\text { no estandarizado }\end{array}$ & $\begin{array}{l}\text { PERFIL DE: } \\
\text { Dublin Core }\end{array}$ & $\begin{array}{c}\text { sí } \\
\text { (por medio de su } \\
\text { clase de } \\
\text { metadatos } \\
\text { PBCoreExtensio } \\
\text { ns) }\end{array}$ & & & en tiempo \\
\hline SMPTE & $\begin{array}{l}\text { desarrollado por: } \\
\text { society of motion } \\
\text { pictures and } \\
\text { television engineers } \\
\text { enlace: } \\
\text { https://www.smpte.org/ } \\
\text { estándar: } \\
\text { smpte st } 335\end{array}$ & & \begin{tabular}{|c|} 
Sí \\
(solo para \\
entornos \\
experimentales, \\
por medio de su \\
clase de \\
metadatos \\
Experimental)
\end{tabular} & & & NO \\
\hline Otros & \multicolumn{6}{|c|}{$\begin{array}{l}\text { - SMEF (BBC) } \\
\text { - MXF Metadata Eschema }\end{array}$} \\
\hline
\end{tabular}


En segundo lugar, se muestra el esquema de metadatos del cual es una extensión o perfil, es decir, cuál es su esquema base. En tercer lugar, se muestra si se permite o no hacer extensiones del esquema de metadatos. En quinto lugar se presentan los perfiles y extensiones de cada esquema de metadatos, de los cuales se han considerado solamente los que describen cualquier contenido de audio, video o audiovisual. En sexto y último lugar, se indica cuáles esquemas pueden describir un segmento del contenido audiovisual ya sea en tiempo o en espacio, en especial, los que permiten la segmentación en tiempo utilizan el mismo conjunto de metadatos destinados a describir todo el recurso para marcar un segmento del mismo, por ejemplo, "género" en PBCore es un metadato de descripción y por lo tanto también será posible establecer un género para un segmento. (La

la jerror! la autoreferencia al marcador no es válida. muestra una comparación entre los anteriores esquemas de metadatos por medio de cinco elementos. en primer lugar, se presenta su creador junto con el nombre del estándar o especificación y su enlace. en este punto es importante aclarar que una especificación es una propuesta de organismos que no ha sido aprobada por algún organismo oficial de estandarización, mientras que un estándar es una tecnología, formato o método ratificado por algún organismo oficial de estandarización, en algunos casos, una especificación puede considerarse un estándar de facto si su uso es extendido y entretanto se ratifica como estándar. 
tabla 1 continua en la siguiente página).

Ahora bien, TVA y P-META no permiten la segmentación en tiempo con la anterior capacidad, por el contrario, limitan los metadatos utilizados para tal fin, por ejemplo, TVA permite la descripción de segmentos únicamente con: título, sinopsis, género, palabras clave, enlaces a material externo relacionado y lista de créditos, sin embargo, tiene la capacidad de agrupar segmentos con un propósito particular o característica similar y asociarles metadatos a los grupos de segmentos y de esta manera posibilita la reestructuración de un flujo audiovisual para proporcionarle al usuario otra forma de interactuar con el contenido (Rey-López et al., 2010). Por otro lado, un modelo de descripción de contenidos de televisión debe ser capaz de representar los siguientes conceptos (ETSI, 2012a):

\section{1.- Un programa simple.}

2.- Un programa con un número de versiones diferentes (por ejemplo: ediciones por sexto/violencia/idioma, corte del director, etc.).

3.- Un programa que ha sido dividido partes para su publicación (por ejemplo: 3 horas de filmación mostradas en 2 partes en diferentes días).

4.- Un programa que es una concatenación de una secuencia de otros programas identificado como un programa agregado.

5.- Una serie de programas que pueden ser ordenados (por ejemplo: episodios en un orden numérico) o desordenados y limitados o ilimitados.

6.- Una colección de series y programas individuales que tienen el mismo concepto, es decir, un show (por ejemplo: todas las series de "Only Fools and Horses" junto con los especiales de navidad).

7.- Una publicación de un programa que puede tener atributos dependientes de la publicación (por ejemplo: una película que se presenta como homenaje a un actor fallecido recientemente, tendría una descripción diferente a la original).

Teniendo en cuenta que la mayoría de las especificaciones y estándares estudiados se enfocan en la descripción de contenidos multimedia para la televisión, la tabla 2 muestra cuáles de los anteriores requerimientos de descripción de contenido cumplen dichos esquemas, basándose en la documentación y sus archivos XSD de definición (un archivo XSD (XML Schema Definition), como su nombre lo indica, describe la estructura de un documento XML. También se conoce como Esquema XML (XML Schema)).

Tabla 2: Esquemas de metadatos vs Requerimientos de descripción de contenido de TV.

${ }^{*}$ Cumplen con el requerimiento pero de una manera limitada.

\begin{tabular}{|c|c|c|c|c|c|c|c|}
\hline Esquema & 1 & 2 & 3 & 4 & 5 & 6 & 7 \\
\hline MPEG-7 & $\checkmark$ & - & - & - & - & - & - \\
\hline TVA & $\checkmark$ & $\checkmark$ & $\checkmark$ & $\checkmark$ & $\checkmark$ & $\checkmark$ & $\checkmark$ \\
\hline P-META & $\checkmark$ & $\checkmark^{*}$ & - & - & $\checkmark$ & $\checkmark$ & - \\
\hline EBUCore & $\checkmark$ & $\checkmark$ & $\checkmark^{*}$ & - & $\checkmark$ & $\checkmark$ & - \\
\hline PBCore & $\checkmark$ & - & - & - & $\checkmark^{*}$ & $\checkmark^{*}$ & - \\
\hline SMPTE & $\checkmark$ & - & - & - & - & - & - \\
\hline
\end{tabular}

Sintetizando lo expuesto anteriormente, la comparación presentada en la tabla 2 evidencia a los esquemas de metadatos que fueron diseñados para el contexto de la televisión y muestra claramente cuáles cumplen con los requerimientos de descripción de contenidos de televisión, de ahí que, el estándar TVA y EBUCore son los más adecuados para cubrir dichos requerimientos. De igual manera, teniendo en cuenta la revisión bibliográfica realizada, se observó que dentro del conjunto de esquemas de metadatos cuyo objetivo principal son los contenidos de televisión, TVA es el más utilizado, especialmente en trabajos de investigación tales como (Hyun-Cheol et al., 2008) (Sotelo et al., 2009) (Blanco-Fernández et al., 2011) (Qingjun et al., 2011) (Young-Guk et al., 2011) entre muchos otros. Sin embargo, a pesar de lo anterior y de que la esencia de TVA es realizar una descripción del contenido que contribuya en el soporte de experiencias personalizadas del televidente, MPEG-7 es el estándar con más influencia en el campo de la marcación de contenidos multimedia (Rey-López, 2009). 
En consecuencia, las normas más adecuadas y utilizadas en televisión para etiquetar contenidos multimedia son TVA y MPEG-7, no obstante, escoger una norma u otra depende del contexto de aplicación, por ejemplo, en algunos casos será preferible la simplicidad de TVA con respecto a la complejidad de la sintaxis de MPEG-7, en otros casos será necesario proveer a los programas de televisión con una información semántica considerable y MPEG-7 lo facilitará (Rey-López et al., 2010), y en otros será preferible implementar la simpleza de la segmentación de TVA en comparación con MPEG-7 porque responderá mejor a las limitaciones físicas y operacionales de la difusión (NoTube, 2010).

\section{CONCLUSIONES}

Como conclusión a la revisión realizada, las comparaciones y caracterización de los esquemas de metadatos adecuados para la marcación de contenido multimedia en el contexto de la televisión, se puede afirmar que los esquemas más aptos para la descripción de este tipo de recursos son TVA y MPEG-7 porque TVA cumple con los requerimientos de marcación de contenido de televisión y MPEG-7, por su parte, es el estándar con mayor influencia en temas de marcación de contenido multimedia.

A pesar de lo anterior, TVA y MPEG-7 tienen características opuestas que pueden representar un pro o un contra de acuerdo a la situación en la que se deseen utilizar y por eso la decisión de implementar uno u otro en el ámbito de la televisión dependerá del contexto de aplicación (noticias, educación, películas, publicidad, todos los contenidos de televisión, etc.). Las características opuestas más claras son: objetivo principal, sintaxis e implementación, y segmentación. En primer lugar, el objetivo de TVA es la definición de experiencias televisivas mientras que el de MPEG-7 es la descripción de recursos multimedia independientemente del entorno del recurso. En segundo lugar, la sintaxis e implementación de TVA es sencilla mientras que MPEG-7 tiene una sintaxis compleja y en consecuencia una implementación engorrosa. En tercer lugar, la segmentación permitida por TVA es en tiempo y por medio de un número limitado de metadatos básicos, mientras que MPEG-7 permite una segmentación en tiempo, espacio y espacio-temporal por medio de gran cantidad de metadatos, por lo tanto es capaz de proporcionar mayor información semántica y describir escenas complejas.

Finalmente es importante resaltar las diferentes relaciones entre los esquemas de metadatos estudiados. PBCore y EBU Core se basan en el mismo esquema Dublin Core (DC), sin embargo, el primero es un perfil mientras que el segundo es una extensión de DC. Aunque P-META sea relativamente actual (2011), EBU Core lo reemplaza permitiendo la implementación de diferentes perfiles y extensiones orientados a aplicaciones particulares, por ejemplo: publicidad. Por otro lado, se encuentra TV-Anytime el cual no tiene un esquema de metadatos base específico, pero sí ha escogido a DDL como su formato de representación de metadatos, utiliza la misma filosofía de esquemas de clasificación y reutiliza tipos de datos básicos definidos por MPEG-7 para construir sus archivos de definición. Finalmente, MPEG-7 al ser el esquema más utilizado para la marcación de contenido multimedia, ha servido de base para el desarrollo de diferentes perfiles de aplicación y extensiones.

\section{REFERENCIAS}

Acevedo C.P., Arciniegas J. L., García X., Perrinet J. (2010). "Proceso de Adaptación de una Aplicación de e-aprendizaje a t-aprendizaje", doi: 10.4067/S0718-07642010000600005, Información tecnológica, vol.21, no.6, pp 27-36, (2010)

Blanco-Fernández Y., López-Nores M., Gil-Solla A., Ramos-Cabrer M., Pazos-Arias J.J., "User-generated contents and reasoning-based personalization: Ingredients for a novel model of mobile TV", doi: 10.1016/j.eswa.2010.10.029, Expert Systems with Applications, vol.38, no.5, ISSN 0957-4174, pp 52895298, Mayo (2011)

CINTEL, "Documento final - Dinámica sectorial, debilidades y retos de la generación de contenidos sobre diferentes plataformas v5.0.1", Bogotá D.C, Marzo (2010)

Delgado J., Llorente S., Peig E., Carreras A. "Metadata and Rights Interoperability for Content Interchange between Producers of TV Programmes". In Proceedings ELPUB2006 Conference on Electronic Publishing, Bansko, Bulgaria, Junio (2006)

European Broadcasting Union EBU, "EBU - TECH 3295, P_META Metadata Library, Specification 2.2", Geneva, Septiembre (2011)

European Broadcasting Union EBU, "P_META", EBU Technology \& Innovation - P-META, (s.f), Recuperado el 18 de junio de 2014 de https://tech.ebu.ch/metadata/p_meta 
European Broadcasting Union EBU, “TECH 3293 EBU Core metadata set (EBUCore), Version 1.5”, Geneva, Abril (2014)

European Broadcasting Union EBU and Association of Television and Radio Sales Houses EGTA, "EBU TECH 3340, Metadata for the file exchange of advertising material (egtaMETA), Specification v1.0", Octubre (2010)

European Telecommunications Standards Institute ETSI, "ETSI TS 102 822-1 V1.3.1 - Broadcast and Online Services: Search, select, and rightful use of content on personal storage systems ("TV-Anytime"); Part 1: Benchmark Features, Technical Specification (2006)

European Telecommunications Standards Institute ETSI, "ETSI TS 102 822-2 V1.4.1 - Broadcast and Online Services: Search, select, and rightful use of content on personal storage systems ("TV-Anytime"); Part 2: Phase 1 - System description", Technical Specification (2007)

European Telecommunications Standards Institute ETSI, ETSI TS 102 822-3-1 V1.8.1 - Broadcast and Online Services: Search, select, and rightful use of content on personal storage systems ("TV-Anytime"); Part 3: Metadata; Sub-part 1: Phase 1 - Metadata Schemas, Technical Specification (2012)

European Telecommunications Standards Institute ETSI, "ETSI TS 102 822-3-3 V1.6.1 - Broadcast and Online Services: Search, select, and rightful use of content on personal storage systems ("TV-Anytime"); Part 3: Metadata; Sub-part 3: Phase 2 - Extended Metadata Schema, Technical Specification (2012)

Feng D.D, Siu W.C., Zhang H.J, Zhang, Hong Jiang "Multimedia Information Retrieval and Management. Technological Fundamentals and Applications". doi: 10.1007/978-3-662-05300-3. Series: Signals and Communication Technology. (2003)

Hyun-Cheol K., Seong Y.L., Joo M.S; Ji H.J.; Han-Kyu L.; Jin-Woo H., "Development of advanced PVR based on TV-Anytime", doi: 10.1109/ICCE.2008.4588028, Consumer Electronics, 2008. ICCE 2008. Digest of Technical Papers. International Conference, pp.1, 2, 9-13 Enero (2008)

International Telecommunication Union ITU-T, "Supplement on IPTV service use cases", ITU-T Y-series Recommendations - Supplement 5, Mayo (2008)

Joint Information Systems Committee JISC, "Metadata Standards and Interoperability", JISC Digital Media I Guides, (s.f), Recuperado el 20 de mayo de 2014 de http://www.jiscdigitalmedia.ac.uk/guide/metadatastandards-and-interoperability.

Joint Information Systems Committee JISC, "Putting Things in Order: a Directory of Metadata Schemas and Related Standards", JISC Digital Media | Guides, (s.f), Recuperado el 10 de septiembre de 2014 de http://www.jiscdigitalmedia.ac.uk/guide/putting-things-in-order-links-to-metadata-schemas-and-related-

standards.

Lapuente M.J.L., "Hipertexto, el nuevo concepto de documento en la cultura de la imagen", Tesis doctoral, Universidad Complutense de Madrid, Departamento de Biblioteconomía y Documentación. España, (2006)

Marcos G. "A Semantic Middleware to enhance current Multimedia Retrieval Systems with Content-based functionalities" Tesis doctoral, Universidad del País Vasco - Euskal Herriko Unibertsitatea, España, (2011)

N. I. S. O. NISO. "Understanding metadata", Technical report, National Information Standards Organization NISO, (2004)

NoTube Project Networks and ontologies for the transformation and unification of broadcasting and the Internet, "D2.1 Requirements analysis", Febrero (2010)

Open IPTV Forum, "Specification Functional Architecture v2.2", Release 2, Mayo (2013)

Public Broadcasting Metadata Dictionary Project PBCore, "Abourt PBCore", About|PBCore, 2011, Recuperado el 19 de mayo de 2014 de http://www.pbcore.org/about/ 
Public Broadcasting Metadata Dictionary Project PBCore, "PBCore Documentation", Documentation|PBCore, 2011, Recuperado el 19 de mayo de 2014 de http://www.pbcore.org/documentation/

Public Broadcasting Metadata Dictionary Project PBCore, "PBCore Elements", Elements|PBCore, 2011, Recuperado el 19 de mayo de 2014 de http://www.pbcore.org/elements/

Peig E., "Interoperabilidad de Metadatos en sistemas distribuidos", Tesis doctoral, Universitat Pompeu Fabra, España, Octubre (2003)

Qingjun W., Zhihong W., "Research into application of TV-anytime standard on digital Televisions", doi: 10.1109/AIMSEC.2011.6011421, Artificial Intelligence, Management Science and Electronic Commerce (AIMSEC), 2011 2nd International Conference on, pp.6318, 6321, 8-10, Agosto (2011)

Rey-López M., "Marco Conceptual y Arquitectura para el Aprendizaje Personalizado a través de Televisión Digital Interactiva", Tesis doctoral, Universidad de Vigo, España, (2009)

Rey-López M., Fernández-Vilas A., Díaz-Redondo R.P, López-Nores M., Pazos-Arias J.J., Gil-Solla A., Ramos-Cabrer M., García-Duque J. "Enhancing TV programmes with additional contents using MPEG-7 segmentation information”, doi: 10.1016/j.eswa.2009.06.053, Expert Systems with Applications: An International Journal archive. vol.37, no.2, Pages 1124-1133, Marzo (2010)

Sotelo R., Blanco-Fernandez Y., Lopez-Nores M., Gil-Solla A., Pazos-Arias J.J., "TV program recommendation for groups based on muldimensional TV-anytime classifications", doi: 10.1109/TCE.2009.4814442, Consumer Electronics, IEEE Transactions on, vol.55, no.1, pp.248,256, Febrero (2009)

The Society of Motion Picture and Television Engineers SMPTE, "Metadata Element Dictionary Structure SMPTE ST 335:2012 Revision of SMPTE 335M-2001", Enero 13 (2012)

TV-Anytime Forum, "WG Metadata (MD)", TV-Anytime Website, 2003, Recuperado el 30 de junio de 2014 de http://www.tv-anytime.org/workinggroups/wg-md.html

Vargas-Arcila A.M., Baldassarri S., Arciniegas J.L, "Propuesta de marcación de contenidos multimedia educativos en entornos de IPTV", In Proceedings of Interactive of Digital TV Workshop of Webmedia 2014 (WTVDI/Webmedia 2014 (XX Simpósio Brasileiro de Sistemas Multimídia e Web)), (2014)

Vivancos-Vicente P.J. "El estándar MPEG-7" InforMAS Revista de Ingeniería Informática del CIIRM", Dep.Legal: MU-2419-2004. ISSN: 1698-884, (2005)

Young-Guk H., Beom-Seok J., Bong-Jin O., Yu-Seok B., Eui-Hyun P., "Effective encoding of TV-Anytime metadata using EXI", doi: 10.1109/ICCE.2011.5722680, Consumer Electronics (ICCE), 2011 IEEE International Conference on, pp.455,456, 9-12, Enero (2011) 